\title{
The Distribution of NCAM in the Chick Hindlimb during Axon Outgrowth and Synaptogenesis
}

\author{
Kathryn W. Tosney,* Michiko Watanabe, $†$ Lynn Landmesser, $\$ 1$ and URS Rutishauser $\dagger$ \\ ${ }^{*}$ Division of Biological Sciences, University of Michigan, Ann Arbor, Michigan 48109; $†$ The Department of Developmental Genetics and \\ Anatomy, Case Western Reserve University, Cleveland, Ohio 44106; and $\ddagger$ Department of Physiology and Neurobiology, \\ 75 N. Eagleville Rd., Rm 416, The University of Connecticut, Storrs, Connecticut 06268 \\ Received August 19, 1985; accepted in revised form November 1, 1985
}

\begin{abstract}
We have determined the distribution and form of the neural cell adhesion molecule (NCAM) in the chick hindlimb from initial axon outgrowth (stage 171 ) until 3 days posthatching by immunohistological staining and sodium dodecyl sulfate-polyacrylamide gel electrophoresis immunoblots. Axons stained intensely for NCAM at all ages, whereas nonneuronal limb components exhibited dynamic changes in staining. Mesenchymal cells in the sclerotome adjacent to the neural tube developed NCAM immunoreactivity in an anterior-posterior sequence which correlated with the sequence of axonal outgrowth. Low to moderate amounts of NCAM were detected within and surrounding presumptive nerve pathways, consistent with a permissive role for NCAM in axon extension, but not with a precise delineation of pathway boundaries. On myotubes immunoreactivity for NCAM remained low from stage 26 to 30 when it increased dramatically in both aneural and control limbs, indicating that its appearance is not triggered by nerve-dependent activity or trophic interactions. The increase was temporally associated with muscle cleavage and may encourage subsequent axon ramification as well as synaptogenesis. Staining remained high on muscle fibers during secondary myotube formation and only declined during the week before hatching when polyneuronal innervation is withdrawn and the mature synaptic pattern becomes stabilized. This loss of muscle NCAM occurred first on fast and then on slow muscle fibers. Together these results suggest that the timing of innervation may be controlled by the muscle, through NCAM expression, but that the subsequent suppression of muscle NCAM may occur as a result of nerve-mediated activity. (c) 1986 Academic Press, Inc.
\end{abstract}

During development, axons in various parts of the peripheral nervous system must extend axons over relatively long distances to reach their appropriate targets; many do so by following highly reproducible pathways (Lance-Jones and Landmesser, 1981b; Lewis et al., 1981; Raper et al., 1983b) with considerable precision (Landmesser, 1978; Lance-Jones and Landmesser, 1981a; Raper et al, 1983a; Hollyday, 1983). The axonal growth cone accomplishes this by responding to a number of guidance cues in the intervening environment and by stopping and forming a synapse once the target is encountered.

In the chick limb, pathway selection and synaptogenesis have been shown to be highly selective (Landmesser and Morris, 1975; Lance-Jones and Landmesser, 1981a; Hollyday, 1983; Tosney and Landmesser, 1985a) and for motoneurons these processes have been analyzed following a variety of experimental perturbations (Lance-Jones and Landmesser, 1980a,b, 1981b; Lewis et al., 1981; Summerbell and Stirling, 1981; Hollyday, 1981; Whitelaw and Hollyday, 1983a,b). These studies have led to the proposal that in the limb there is a dual system of axon guidance features. One is a relatively nonspecific set of "public" pathways down which all axons prefer

\footnotetext{
${ }^{1}$ To whom requests for reprints should be sent.
}

to grow (such as the substrate pathways proposed by Singer et al. (1979) and Katz and Lasek (1979)). It is these pathways that are believed to create the stereotyped anatomical nerve pattern (Lewis et al., 1981; LanceJones and Landmesser, 1981b; Tosney and Landmesser, 1984). Such "public" pathways appear to be sensed by all growth cones, since even foreign motoneurons will follow them (Hamburger, 1939; Hollyday, 1981; LanceJones and Landmesser, 1981b; Straznicky, 1983). A second set of specific cues is required to explain the ability of different subpopulations of motoneurons to select their individually appropriate pathways or targets (Lance-Jones and Landmesser, 1980b, 1981a,b). Both forms of guidance can contribute to the final precision of innervation; the substrate pathways bring axons into regions where they then can respond to relatively local specific signals (Lance--Iones and Landmesser, 1981b; Summerbell and Stirling, 1981).

Although many different mechanisms have been postulated to contribute to selective axon guidance and synapse formation, adhesive interactions between the growth cones and their environment have always been assigned a central role (Letourneau, 1975; Bray, 1982; Bentley and Keshishian, 1982; Nardi, 1983; Silver and Rutishauser, 1984; Rentley and Caudy, 1983; Berlot and 
Goodman, 1984). In the chick optic system, adhesive interactions between retinal ganglion cell growth cones and glial end-feet have been shown to be mediated at least in part by a neural cell surface adhesion molecule, NCAM, and thereby to contribute to the guidance of optic axons to the tectum (Silver and Rutishauser, 1984).

In addition to axon-glial interactions, NCAM has been shown to promote adhesion not only between neurons (Rutishauser et al, 1978; Rutishauser and Edelman, 1980) but also between neurons and myotubes (Grumet et al., 1982; Rutishauser et al., 1983).

In view of the potential contribution of NCAM-mediated adhesion to both axon guidance and target interactions, we have used histological staining methods to define the spatial and temporal expression of NCAM in the chick hindlimb from the time of initial axon outgrowth through hatching. In this preparation the distribution of NCAM appeared at least in part to define both a system of public pathways and also to modulate the interaction of axons with their targets. A brief report of these findings has appeared previously (Tosney et al., 1985).

\section{MATERIALS AND METHODS}

\section{Immunostaining}

Staining of tissue was carried out with a mouse monoclonal antibody that recognizes an extracellular polypeptide-dependent determinant on chicken NCAM (Thanos et al., 1984). This antibody reacts well with the 120-, 140-, and 180-Da forms of the NCAM polypeptide regardless of their degree of sialylation (U. Rutishauser and M. Watanabe, unpublished observations). The antibody is specific for NCAM as judged by its ability to isolate this molecule in pure form from a Nonidet P40 extract of whole brain tissue.

White leghorn chicken embryos of various ages were fixed in $3.7 \%$ formaldehyde in phosphate-buffered saline (PBS) at $\mathrm{pH} 7.2$ for 1-2 $\mathrm{hr}$. They were then washed in three changes of PBS for 30 min each, impregnated with $20 \%$ sucrose for $3-12 \mathrm{hr}$ depending on the age of the tissue, and then frozen in Tissue-Tec OTC compound. Sections were cut at a thickness of 10-12 $\mu \mathrm{m}$ and dried for at least $30 \mathrm{~min}$ on gelatin-coated slides. The sections were then stained by sequential incubation in (a) $10 \mu \mathrm{g} /$ $\mathrm{ml}$ monoclonal anti-NCAM in PBS containing $1 \mathrm{mg} / \mathrm{ml}$ bovine serum albumin for $1 \mathrm{hr}$; (b) three 15 -min rinses in PBS; (c) a 1:200 dilution of fluorescein isothiocyanate (FITC)-labeled goat anti-mouse immunoglobulin G (IgG) (Sigma) or a 1:150 dilution of peroxidase-labeled goat anti-mouse IgG (Sigma) for 30 min, (d) three 15-min rinses in PBS; (e) in the case of the peroxidase-labeled second antibody, sections were then reacted with a 0.25 $\mathrm{mg} / \mathrm{ml}$ solution of diaminobenzidine in Tris buffer con- taining $0.005 \%$ hydrogen peroxide for $15-30 \mathrm{~min}$ followed by two 15-min rinses in PBS. The stained sections were mounted in glycerol and observed by bright-field and Nomarski optics in the case of peroxidase-labeled second antibody, or epifluorescence in the case of the FITC-labeled second antibody. At least four embryos were observed for each stage using the staging criteria of Hamburger and Hamilton (1951). For controls adjacent sections were stained with either a monoclonal antibody against Drosophila yolk or the $1^{\circ}$ or $2^{\circ}$ antibody was omitted. Such controls exhibited virtually no staining (see Fig. 1).

It should be noted that in all cases where the intensity of NCAM staining was compared at different times or different regions, the two sections to be compared were incubated at the same time in the same antibody-containing solutions for at least two embryos. They were photographed at the same exposure and printed under identical conditions. The differences that are described were obvious and reproducible from embryo to embryo.

Since NCAM is a cell-surface membrane protein, changes in the intensity of immunostaining in our tissue sections could have been caused by either a change in the amount of NCAM per unit area of membrane, or a change in the amount of membrane per unit cross-sectional area. We therefore only compared regions in which membrane packing density was similar. Furthermore, the major intensity change we observed was resolvable at the level of individual myotube and muscle fiber, and could not therefore be explained by changes in membrane packing density.

Another factor that could affect staining is the availability of different epitopes on the NCAM molecule. Given that there are multiple forms of NCAM, the availability of different epitopes might vary between tissues and different developmental stages. However, we have carried out our study with a monoclonal directed against the relatively invariant extracellular portion of the molecule, minimizing this potential problem.

To determine the course of NCAM expression in limbs in the absence of nerves, the lumbosacral neural tube was removed from 30 stage 16-17 embryos under sterile conditions (see Lance-Jones and Landmesser, 1980a,b, for additional details of the operative procedure). The eggs were resealed and returned to the incubator until stages 30-36. Completeness of neural tube removal was confirmed by dissection and by electrical stimulation of cord anterior and posterior to the deleted region of cord. Contractions to stimulation were obtained in only a few cases and were confined to the most posterior ventral thigh region. Some of the embryos were frozen and stained with anti-NCAM as described above. In others, aneural muscle was dissected for the immunoblot procedure described below. 
NCAM staining was correlated with the distribution of fast and slow muscle fibers, determined in a separate study using myosin ATPase staining (Vogel and Landmesser 1986; see also McLennan, 1983). There are striking regional differences in the distribution of fast and slow muscle fibers in most muscles of the chick thigh (McLennan, 1983; Vogel and Landmesser, 1986), facilitating this comparison.

\section{Electrophoretic Analysis of Tissue NCAM}

Brain, retina, and breast muscle from 10- to 11-dayold embryonic chicks (stages 35-36) were dissected on the day of sample preparation for sodium dodecyl sulfate-polyacrylamide gel electrophoresis (SDS-PAGE; Laemmli, 1970) and stored frozen in buffered Tyrode's at $-20^{\circ} \mathrm{C}$. The sciatic plexuses of embryos, together with distal spinal nerves and proximal sciatic nerve trunk, were dissected with sharpened tungsten needles, and freed of adherent mesenchyme at three different stages (stages 28-30, 30-33, and 40). Nerve-free regions of muscle were dissected in a similar manner. In other embryos in which the neural tube had been previously removed, all muscle tissue was dissected free from cartilage and overlying connective tissue.

All analytical procedures were carried out on ice (0$4^{\circ} \mathrm{C}$ ) except where indicated. Tissues were pelleted, solubilized in 10 volumes of Nonidet-P40 (NP-40) buffer $\left(0.5 \% \mathrm{NP}-40,0.8 \% \mathrm{NaCl}, 0.02 \% \mathrm{KCl}, 0.02 \% \mathrm{KH}_{2} \mathrm{PO}_{4}\right.$, $0.015 \% \mathrm{Na}_{2} \mathrm{HPO}_{4}$ by sonication and incubated overnight with rotary mixing with beads (Bio-Rad, Affi-Gel 10) coupled to the anti-NCAM monoclonal antibody described above. The beads were then washed five times with NP-40 buffer and boiled for $3 \mathrm{~min}$ in an equal volume of $2 \times$ sample buffer $(0.0625 M$ Tris buffer at $\mathrm{pH} 6.8$, $2 \%$ SDS, $5 \%$ 2-mercaptoethanol, $10 \%$ glycerol, $0.001 \%$ bromphenol blue). The proteins were separated on $7 \%$ polyacrylamide gels by SDS-PAGE (Laemmli, 1970) and transferred to nitrocellulose filters (Schleicher and Schuell, $0.45 \mu \mathrm{m}$ ) by electrophoresis (Hoefer, TE 42 Transphor with the power supply TE 51 set at $0.5 \mathrm{amps}$ for $12 \mathrm{hr}$ ). The NCAM on the nitrocellulose was detected using an indirect radioimmune staining procedure (Towbin et al., 1979) with modifications described by Johnson et al. (1984). Briefly, the filters were incubated with rabbit antisera directed against embryonic chick brain NCAM (Thanos et al., 1984), washed extensively, and then incubated with [ $\left.{ }^{125} \mathrm{I}\right]$ goat anti-rabbit IgG (New England Nuclear, $94 \mu \mathrm{Ci} / \mathrm{ml}$ ). The bound radioactivity was detected using X-Omat AR X-ray film with an enhancing screen (Swanstrom and Shank, 1978).

\section{RESULTS}

The monoclonal antibody against NCAM used in these studies reacts with all the described polypeptide and glycosylated forms of this molecule. This antibody stained neurons intensely in the chick limb throughout the developmental ages studied (stage 18-3 days posthatching). It also stained muscle and premuscle mesenchyme in a dynamic age-dependent manner as described below.

The range and specificity of staining for NCAM is illustrated in Fig. 1, which represents a transverse section through the adductor region of a stage $32 \mathrm{limb}$. At this stage, NCAM staining occurs on both the developing myotubes as well as the intramuscular nerve branches (Fig. 1A, arrows) which at this time are beginning to ramify within the individual muscles (Tosney and Landmesser, 1985a). An adjacent control section in which the primary monoclonal antibody was directed against an irrelevant antigen, Drosophila yolk, showed no staining (Fig. 1B). Staining was also absent when the primary anti-NCAM antibody or the secondary antibody was omitted (not shown). Figure 1C represents a more distal section through the same muscle that has been stained by a mouse monoclonal directed against a neurofilament component (kindly supplied by $\mathrm{H}$. Tanaka). By comparing Figs. $1 \mathrm{~A}$ and $\mathrm{C}$, it can be seen that the intensely stained profiles visualized within the muscle by the NCAM antibody are indeed intramuscular nerve branches.

The main events in axon outgrowth will first be briefly described as a context for our observations. Between embryonic stages 18 and $23 \frac{1}{2}$, axons from chick lumbosacral motoneurons grow out of the spinal cord and collect at the base of the limb forming the anterior crural plexus and posterior sciatic plexus (Lance-Jones and Landmesser, 1981a; Hollyday, 1983; Tosney and Landmesser, 1985b). Sensory axons emerging from the dorsal root ganglia join with the motor axons at the limb base after a slight delay (Tosney and Landmesser, 1985a; Landmesser and Honig, 1986). Following a "waiting period" of approximately $24-48 \mathrm{hr}$ in the plexus region, axons invade the limb bud and (by stage 24) make a highly specific choice projecting down either the dorsal or ventral major nerve pathway. Subsequently (stages 26-27), specific groups of axons diverge from these major nerve trunks forming cutaneous and muscle nerves (Lance-Jones and Landmesser, 1981a; Honig, 1982; Hollyday, 1983; Tosney and Landmesser, 1985a,b). Thus by stage 27 the highly specific projection pattern of motor and sensory neurons has been formed.

\section{NCAM Expression along the Spinal Nerve Pathway}

Chick lumbar motor axons first leave the cord at stages 18-19 and grow rapidly through the sclerotomal portion of each somite to collect at the base of each myotome. There is an obvious anterior-to-posterior progression in initial outgrowth so that by approximately stages 19- 

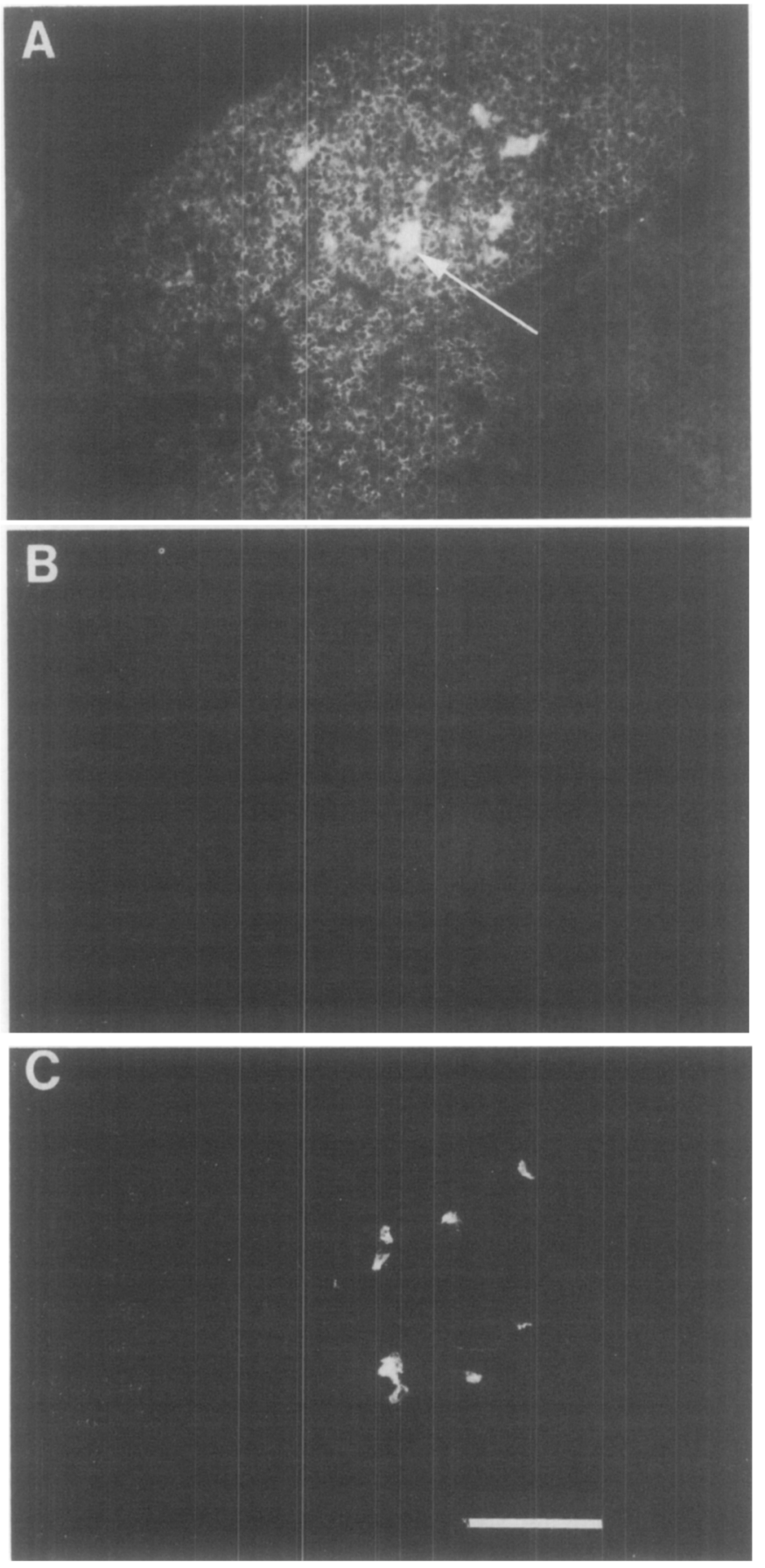

FIG. 1. Specific staining for neural cell adhesion molecule (NCAM) on embryonic nerve and muscle. (A) Transverse 10- $\mu \mathrm{m}$ section through a stage 32 adductor muscle stained with the FITC-conjugated monoclonal antibody against NCAM. Intramuscular nerve bundles (arrow) stain intensely. In addition, the surface of most myotubes stain with moderate intensity. (B) A control adjacent section in which the primary antibody was a monoclonal directed against Drosophila yolk exhibits no staining. (C) A more distal section from the same muscle in which the primary antibody was directed against a neurofilament component, stains only the intramuscular nerve branches. Calibration bar $=100 \mu \mathrm{m}$.
20 the more anterior lumbosacral spinal nerves (LS 1, 2 , and 3) have reached the plexus region, while more posterior ones (LS 7 and 8) have yet to exit from the cord (Tosney and Landmesser, 1985b). Axons normally appear to grow only through the anterior half of each somite. Following embryonic surgery to alter somite position with respect to the cord axons still grow through the original anterior portion of each somite (Keynes and Stern, 1984).

The patterns of staining for NCAM were therefore determined in several stage 18-19 embryos, and a representative example is shown in Fig. 2. In this embryo at the level of LS 3 (Figs. $2 A$ and $B$ ), there was definite NCAM immunoreactivity on both axonal profiles and on mesenchyme cells in the sclerotome region that lay along the course of the prospective nerve pathway. There was also intense staining along the inner surface of the myotome which is shown more clearly in Figs. $2 \mathrm{C}$ and $\mathrm{F}$.

A more posterior section from the same embryo at the level of LS 5 (Figs. 2C and D) revealed moderately stained mesenchyme cells and a few intensely staining profiles (straight arrow) which appear to be the first axons to emerge from the neural tube. At yet more posterior levels (Figs. 2F and G) no axons have emerged although an intensely stained cluster of cells occurred at the most lateral margin of the spinal cord. The sclerotome cells immediately adjacent to the spinal cord at this level exhibited weak staining that was clearly less intense than that at more anterior levels. There is a decline from anterior to posterior in the number of sclerotome cells staining for NCAM as well as the intensity of staining which is similar to the temporal pattern of axon emergence from the cord.

Since the anterior portion of each somite has been shown to be a preferred substratum for axon outgrowth (Keynes and Stern, 1984) we determined if there was a differential distribution of cells which stained intensely for NCAM between the anterior and posterior portions of each somite. A sagittal section from a stage $17 \frac{1}{2}-18$ embryo through a series of lumbar somites close to the neural tube (Fig. 3A) shows distinct clusters of intensely staining profiles in the anterior half of each somite. The number of these profiles and their maximal distance from the spinal cord decreased from more anterior to more posterior somites.

Since stage 17-18 is earlier than axonal outgrowth had been reported at this level based on orthograde horseradish peroxidase (HRP) labeling (Tosney and Landmesser, 1985a,b) we considered that these profiles (shown at higher magnification in Figs. 3B and C) might be part of a nonneuronal substrate pathway for axons. However, preliminary studies (L. Landmesser and $\mathrm{H}$. 
Tanaka, unpublished observations) in which adjacent sections were stained with anti-NCAM or with antibodies directed against either a neurofilament or a motoneuron specific cell surface antigen (Tanaka and Obata, 1984) revealed that staining with these antibodies was essentially coextensive. Specifically, NCAM immunoreactivity was not observed more distally than the immunoreactivity for either of the other antibodies. This strongly suggests that the profiles which stain intensely for NCAM are the earliest axons to leave the spinal cord, and that enhanced NCAM immunoreactivity does not appear to delineate the paths that they will follow.

We also observed low but distinct staining of other cells in the sclerotome (see Figs. 2A-D). This staining was relatively broad along the dorso-ventral axis and would appear to encompass the trajectory taken by motoneurons in the limb (Fig. 2D, curved arrow) as well as the trajectory taken by the small number of motoneurons in each segment that diverge from the spinal nerve pathway to innervate the myotome (Tosney and Landmesser, $1985 \mathrm{~b}, \mathrm{c})$. We did not observe any difference in intensity of this staining between the anterior or posterior half of each somite (Figs. 3B and C), but, as noted above, there was a clear diminution of the intensity of staining along the anterior-posterior axis, and at stages $17 \frac{1}{2}$, staining at posterior lumbar levels was weak to absent (Figs. 2F and G). This low-level background staining was confined to each somite, the intersomitic regions which have been shown to contain fibronectin (Rogers et al., 1983, 1984) being devoid of NCAM immunoreactivity (Figs. 3A-C). Similar low levels of NCAM immunoreactivity were found in the plexus region at these stages (17-19) (Figs. 2C and E) and in the limb mesenchyme just distal to it (not shown). There was therefore no obvious difference in staining for NCAM between plexus and limb regions during the period when axons appear to delay their advance and wait in the plexus (stages 18 $\frac{1}{2}-23$ ) (Tosney and Landmesser, 1985b).

\section{NCAM Expression at the Time Axons Invade} the Limb Bud

Motor axons stained intensely for NCAM during the period that axons grow from the plexus region into the limb to form the major nerve trunks and muscle nerves (stages 24-26). In contrast, only weak staining was observed on mesenchyme cells and early forming myotubes that surround the axons. As shown in the stage $25 \mathrm{em}-$ bryo in Fig. 4B, the main crural nerve trunk just distal to the crural plexus stained intensely. These neurites, followed in serial sections to their termination sites in the dorsal thigh (Fig. 4C), displayed uniform NCAM immunoreactivity throughout their length. More distal sections did not reveal any enhanced staining for NCAM in advance of the nerve trunk (Fig. 4D). Although there was moderate to weak staining for NCAM throughout transverse limb sections, there was no obvious pattern of more intense staining delineating the prospective major nerve trunks (indicated by arrows in the low magnification cross section of the limb bud shown in Fig. 4A).

A view closer to the spinal cord and just proximal to the point where the spinal nerves converge to form the plexus is shown in Fig. 5A for a stage 27 embryo. The ventral roots and spinal nerves just ventral to the moderately stained dorsal root ganglia have intense NCAM immunoreactivity as does the myotomal portion of the somites (dorsally), which are by now differentiating into axial musculature.

When we examined the spinal nerves in stage 25-27 embryos just distal to the point where sensory and motor components join, we consistently noticed that the dorsal part of the nerve, which contains primarily sensory axons, stained less intensely than the ventral portion of the nerve, which contains motor axons (Fig. 5C). Since NCAM is localized in the axolema, differences in axonal packing density could contribute to this differential staining. However, staining with a monoclonal antibody to a cell surface antigen common to both sensory and motor axons (Tanaka and Obata, 1984) does not result in similar differences in staining intensity (L. Landmesser, unpublished observations). Furthermore, at these stages dorsal root ganglia also exhibit weaker staining than the lateral motor column (Fig. 4B; see also Thiery et al., 1982), although both have a similar cell packing density. Thus, it appears that sensory axons at these stages have less NCAM on their surfaces than motor axons.

\section{The Onset of NCAM Expression in Muscle}

Muscle nerves are formed by divergence of axons from major nerve trunks at stage 26 . These muscle nerves penetrate the differentiating primary muscle masses which initially consist of condensed mesenchyme and by stages $26 \frac{1}{2}-27$ form some functional synapses (Landmesser, 1978; Lance-Jones and Landmesser, 1981a). However, there is a marked delay in axonal ramification within the differentiating muscles. Orthograde HRP labeling revealed that most motoneuron growth cones and termination sites were found within these major muscle nerves or in muscle regions immediately adjacent to them, as late as stages $28-29$. Only at stage 30 did axons appear to ramify within the differentiating muscle to any extent (Tosney and Landmesser, 1985a). This observation has been confirmed by using a neurofilament antibody (L. T. Landmesser, unpublished observations). 

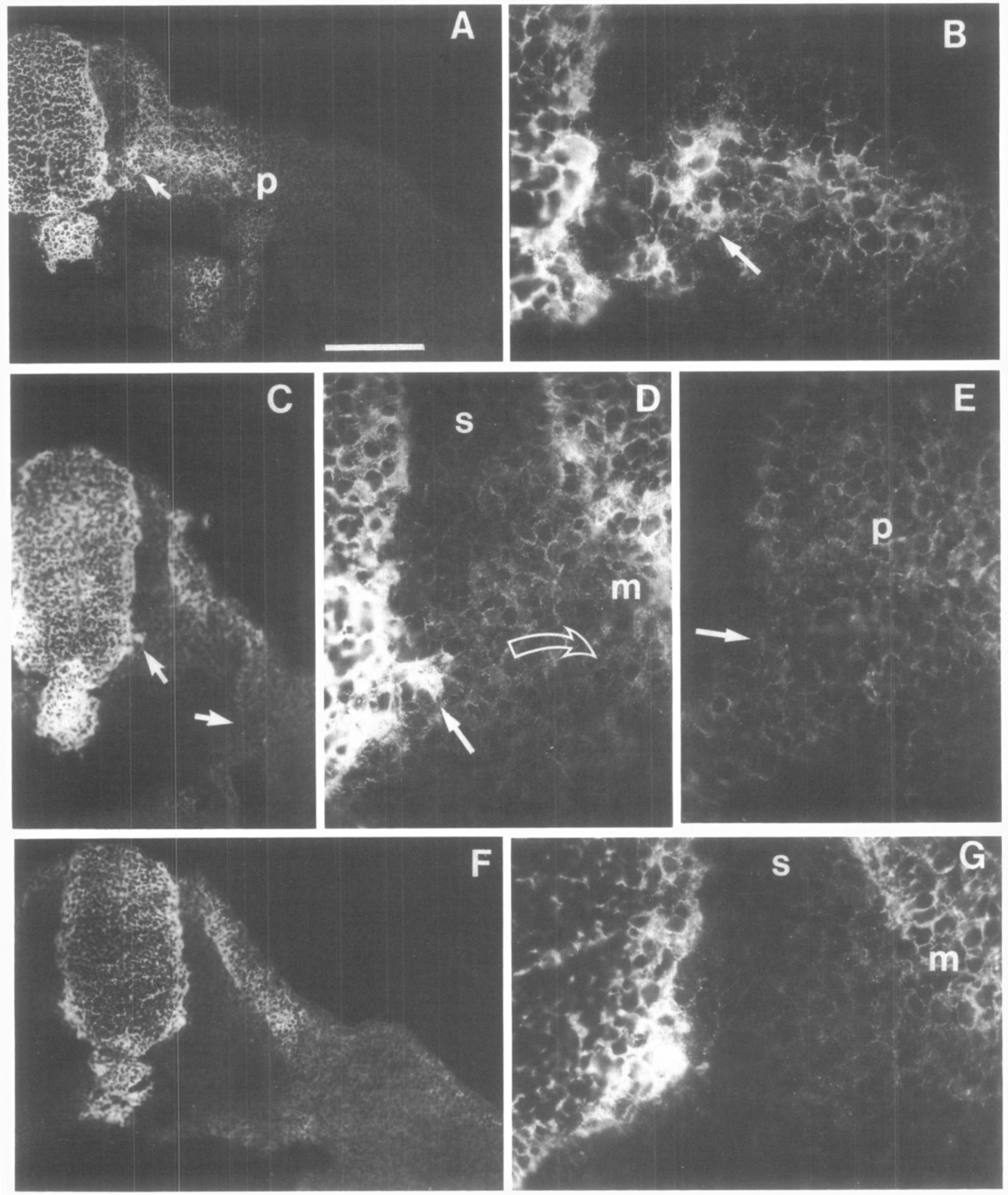

FIG. 2. NCAM distribution adjacent to the neural tube at the time of initial axon outgrowth. Transverse sections through the lumbosacral region of a stage 19 embryo show the location of staining for NCAM in the region surrounding the neural tube. (A) A transverse section through the anterior portion of the lumbosacral cord (LS 3-4) shows intense staining of mesenchyme-like cells (arrow) extending through the sclerotome from the neural tube to the plexus region (p). Staining is weak in the sclerotome adjacent to the neural tube both dorsal and ventral to this intensely staining band of cells which lies in the position of the spinal nerve pathway. (B) The intensely stained region near the neural tube (arrow, A) is shown at higher magnification. (C) A more detailed caudal section through the same embryo (LS 5-6) shows much less intense staining in the sclerotome except at the level of the future ventral root (arrow) where an intensely stained profile extends from the neural tube. (D) At higher magnification weak staining can be seen on cells in the sclerotome between this profile and the myotome (along the trajectory of the future spinal nerve). Intense staining is also observed on the myotome (m) clearly delineating this structure from the adjacent 

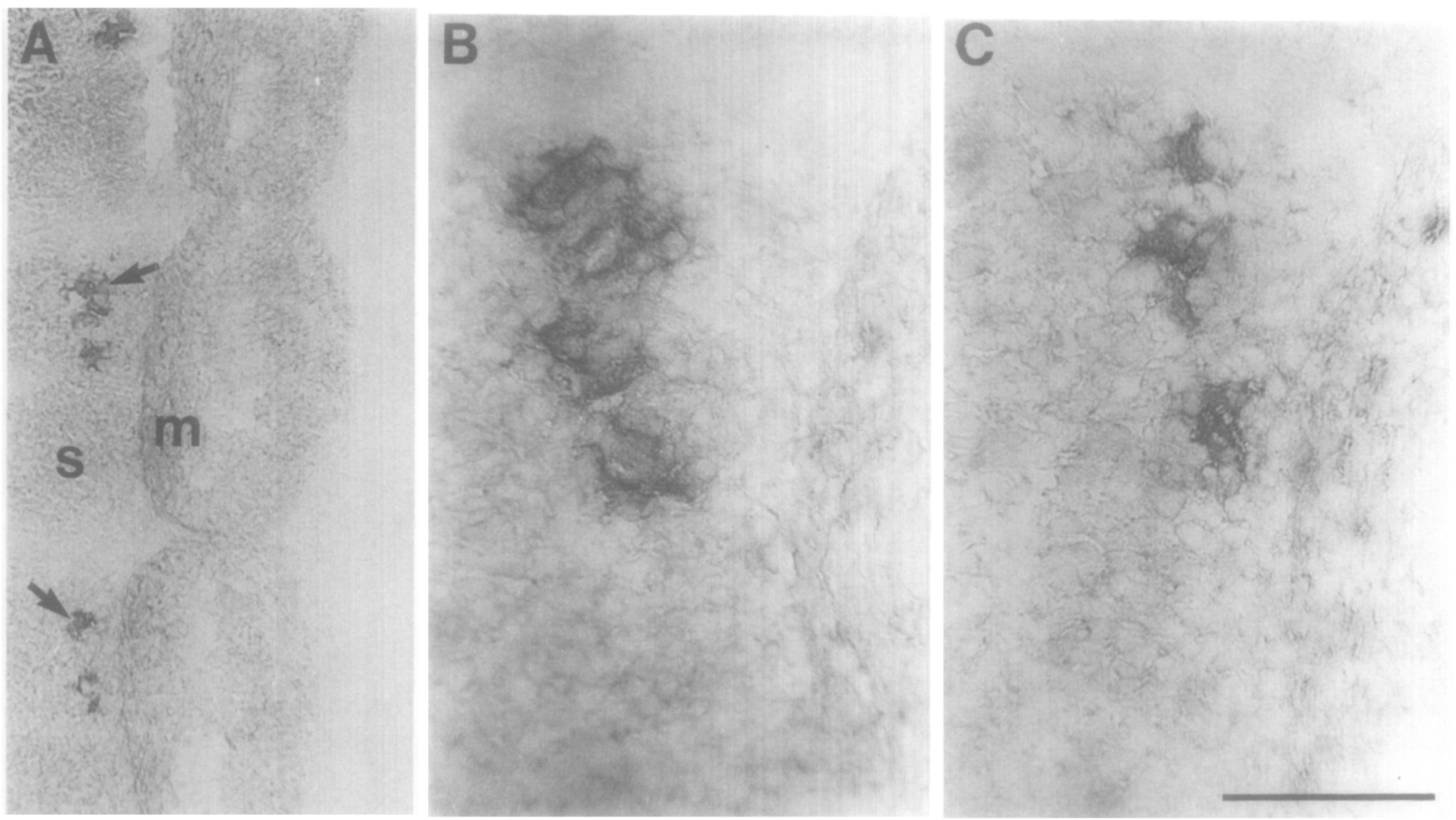

FIG. 3. Distribution of NCAM in the somites prior to axon outgrowth. Sagittal sections through a series of lumbar somites at stages $17 \frac{1}{2}-18$ stained for NCAM in which the secondary antibody was conjugated to peroxidase, so that immunoreactivity for NCAM appears dark. (A) A sagittal section through three somites (anterior up, dorsal to the right) shows staining for NCAM in both the myotome (m) (compare with Figs. 2F, D, and G) and sclerotomal (s) portion of each somite, intersomitic regions staining only weakly. In addition to this moderate staining which appears essentially uniform across the a-p extent of each somite in both the sclerotome and myotome, intensely stained profiles (arrows) are located in the anterior portion of each sclerotome. The number of such profiles decreases in the more posterior somites. These intensely staining profiles are shown at higher magnification for an (B) anterior and a (C) more posterior somite (anterior up, dorsal to the right). Aside from this staining there was no obvious difference in intensity of immunoreactivity for NCAM between the anterior and posterior portions of each somite. Calibration bar $=40 \mu \mathrm{m}$ for $\mathrm{B}$ and $\mathrm{C}$, and $200 \mu \mathrm{m}$ for $\mathrm{A}$.

In the present study we observed a temporal correlation between this ramification and the onset of intense staining for NCAM on differentiating myotubes. Figure 6 shows NCAM immunoreactivity for the iliofibularis and posterior iliotibialis muscles, at stages 28 and 30 . At stage 28, these two muscles have not yet fully cleaved (their presumptive border is marked by an arrow in Fig. $6 \mathrm{~A})$ and there is only weak to moderate staining for NCAM on myotubes. The myotubes with most intense staining were located in the region surrounding the peroneal trunk of the sciatic which abuts against the medial surface of the iliofibularis. It is in fact in this region that most nerve terminals are found at this stage following orthograde labeling with HRP (Tosney and
Landmesser, 1985a). In contrast, by stage 30 (Fig. 6B), both muscles have fully cleaved and myotubes throughout them stain intensely for NCAM. The regions between muscles have only weak staining for NCAM. All muscles exhibited intense staining for NCAM by stages $30-31$; we did not notice any marked temporal differences in the increase in staining intensity between different muscles, including those that develop into either fast or slow muscles.

Staining for NCAM continues to become more intense until by stage 34 (Fig. 7A) it brightly outlines each myotube. By this time many motoneurons have interacted with the myotubes, forming functional synapses (Landmesser and Morris, 1975; Bekoff, 1976; Landmesser,

sclerotome. Staining for NCAM is especially weak in the dorsal region of the sclerotome. (E) A higher magnification view of the plexus region (indicated by small distally pointing arrow in (C)) shows weak to moderate staining for NCAM on most cells in this plexus region where axons will undergo a waiting period for some $48 \mathrm{hr}$ before continuing into the limb. (F) A more posterior section through the same embryo shows very weak NCAM staining in the sclerotome at this level and stage. (G) A higher magnification view showing sclerotome (s) and myotome (m). Calibration bar $=200 \mu \mathrm{m}$ in $\mathrm{A}, \mathrm{C}$, and $\mathrm{F}$, and $50 \mu \mathrm{m}$ in $\mathrm{B}, \mathrm{D}, \mathrm{E}$, and $\mathrm{G}$. 

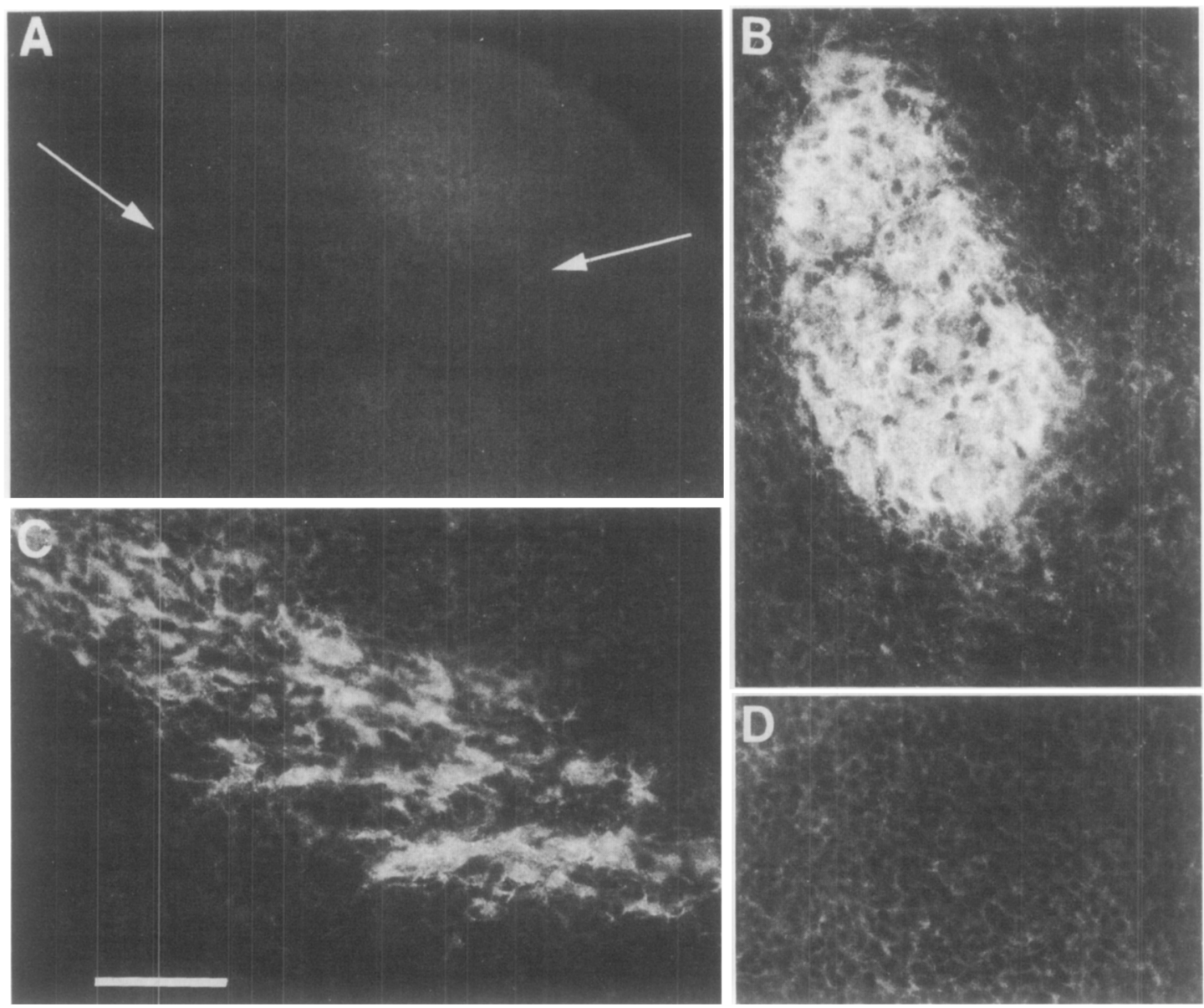

FIG. 4. The distribution of NCAM in the limb bud during invasion of axons. (A) A low magnification transverse section through a stage $24 \frac{1}{2}$ limb bud just distal to the nerve front. There is weak staining for NCAM in some mesenchymal regions but there is no enhanced staining at the sites (arrow) of the future crural and sciatic nerve trunks. Anterior right, dorsal up. (B) A more proximal section through the anterior part of the limb bud showing intense staining of the portion of the femoral nerve trunk pathway that already contains axons. (C) A more distal section reveals the most distal extent of this femoral nerve trunk, showing intense staining for NCAM on axons but only weak staining on surrounding mesenchyme cells. (D) Similarly, the mesenchyme immediately distal to the tip of the femoral nerve has only weak staining. Calibration bar $=500 \mu \mathrm{m}$ in $\mathrm{A}$ and $100 \mu \mathrm{m}$ in $\mathrm{B}, \mathrm{C}$, and D.

1978), and the period of motoneuron cell death is underway (Hamburger, 1975; Chu-Wang and Oppenheim, 1978). In order to determine whether the increase in NCAM immunoreactivity is triggered by the nerve, we created aneural limbs by removing the entire lumbosacral cord at stages 17-18 (prior to axon outgrowth). We found, as shown by the stage 34 aneural muscle of Fig. 7B, that NCAM immunoreactivity increased over the same time course and was not noticeably different from controls. Therefore the increase in NCAM on myotubes does not appear to be triggered by a neural trophic factor or by functional activity of myotubes.

\section{Decreased NCAM Expression in Muscle around Hatching}

Staining for NCAM became intense on muscle fibers during the period when motor axons are forming numerous synapses. Although NCAM expression continues on mature neurons, it declines markedly in mature muscle when nerve-muscle relationships have stabilized (Couvalt and Sanes, 1985; Rieger et ul., 1985). However, in all vertebrate muscles, there is an intervening period of considerable plasticity, during which motor axons make and break connections (see Van Essen, 1982, for review). This period of polyneuronal innervation followed by subsequent withdrawal of supernumerary inputs takes place in the first week after birth in rats (Brown et al., 1976; Thompson, 1983) and around the time of hatching in chicks (Bennett and Pettigrew, 1974; Srihari and Vrbova, 1978; Pockett, 1981). Since changes in NCAM levels might be implicated in this process, we decided to determine when NCAM levels decreased in the chick.

We found that NCAM immunoreactivity on myotubes remained high until stages $39-41$ on all muscles. The stage 39 posterior iliotibialis muscle in Fig. 8A illustrates 

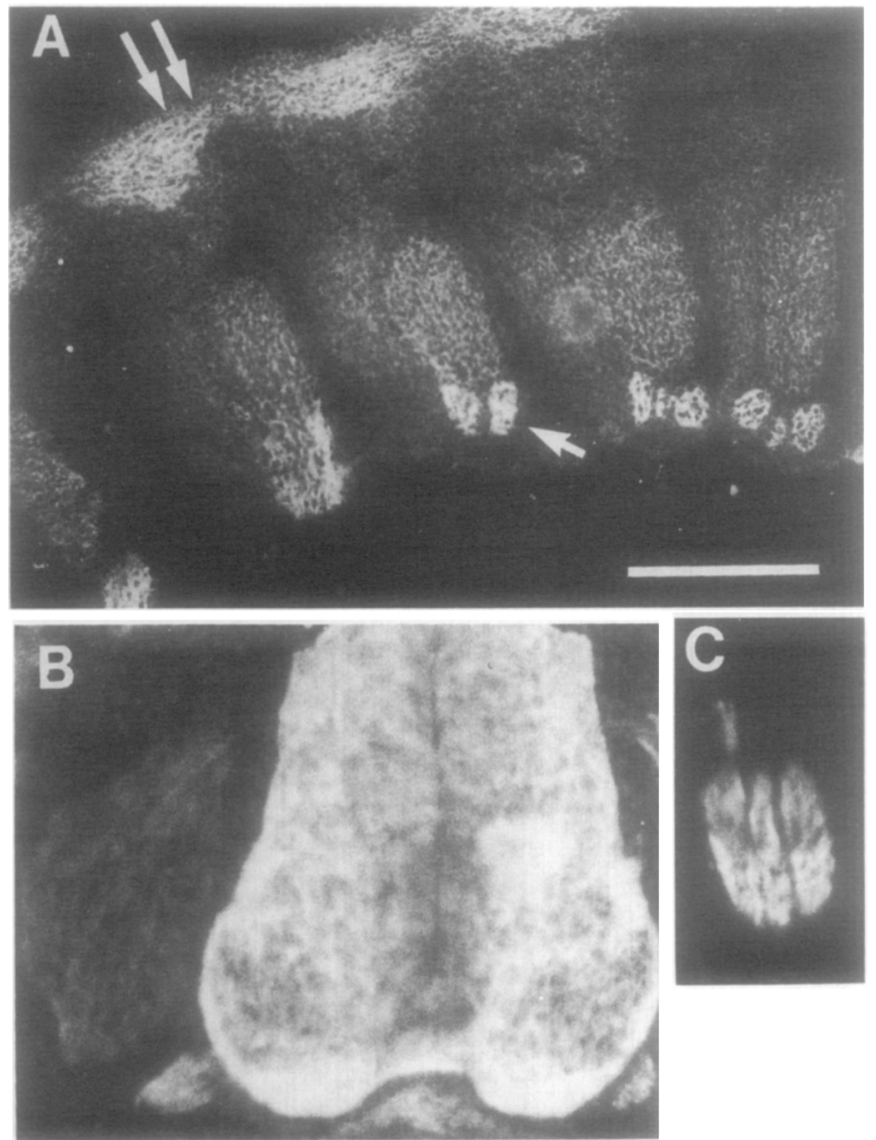

Fig. 5. NCAM expression on sensory and motor neurons at stage 27 during the period of muscle nerve and initial synapse formation. (A) A sagittal section through a series of lumbar dorsal root ganglia (DRGs) shows intensely stained ventral roots (arrow) and axial musculature (double arrow). Cell bodies in the DRGs stain moderately. Anterior right, dorsal up. (B) A transverse section through the spinal cord at this level indicates intense NCAM immunoreactivity in the spinal cord including the lateral motor column but only weak staining in the adjacent DRG. (C) Cross section through a proximal spinal nerve, showing that the dorsal (up) part of spinal nerve, which contains predominatly sensory fibers, stains less intensely than the ventral, predominately motor portion (Calibration bar $=200 \mu \mathrm{m}$ in $\mathrm{A}, 450 \mu \mathrm{m}$ in $\mathrm{B}$ and $\mathrm{C}$ ).

this point. However, from stage 42 on, staining declined, and by 3 days posthatching, was essentially absent from this muscle (Fig. 8B) except for preterminal neurites and synapses (see also Couvalt and Sanes, 1985; Rieger et al., 1985). A cross section of nerve lies adjacent to the muscle and reveals the very intense NCAM immunoreactivity of the axons. The few intensely staining profiles in the muscle appear to be axons or nerve terminals. The decline in NCAM staining is thus temporally correlated with the withdrawal of polyneuronal innervation and stabilization of the adult synaptic pattern, which in the ambiens of the chick thigh, and presumably in other thigh muscles as well, occurs between stage 42 and hatching (Pockett, 1981).
It was immediately apparent that unlike its onset, the decline in staining for NCAM did not occur synchronously in all muscles. By 3 days posthatching, moderate to weak staining for NCAM was seen on the surface of most myotubes in predominantly slow muscles, but was reduced or absent in fast muscles. Figure 9 illustrates this point by showing the boundary between the predominantly slow external adductor and predominantly fast internal adductor (Vogel and Landmesser, 1986) at 1 day prior to hatching (stages 44-45) and 3 days after hatching. Although staining for NCAM is more intense
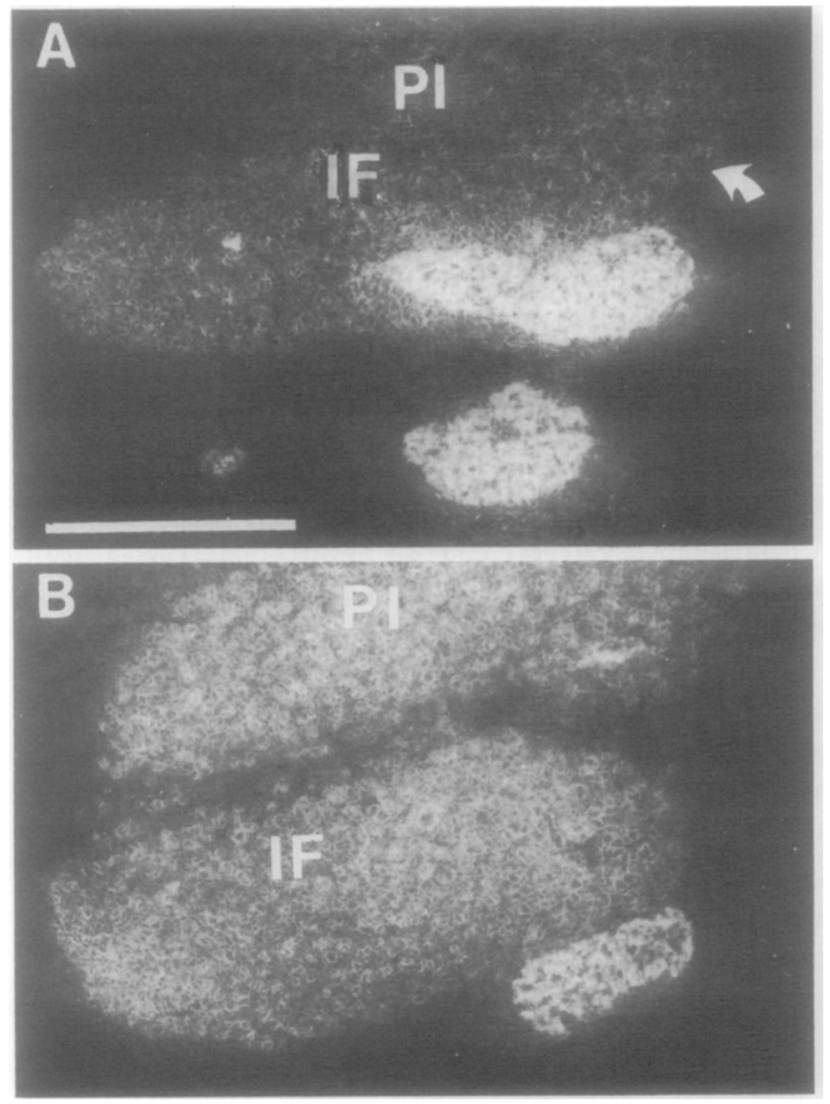

FIG. 6. The onset of enhanced NCAM expression on developing myotubes. (A) A transverse section through the thigh of a stage 29 embryo shows only weak NCAM immunoreactivity on developing myotubes in the posterior iliotibialis (PI) and iliofibularis (IF) muscles. At this stage the muscles are not fully cleaved (arrow indicates presumptive boundary), and motor axons have not significantly ramified within the developing muscles. The two main trunks of the sciatic nerve stain intensely, the dorsal or peroneal trunk abutting the IF muscle. Intensely stained myotubes are restricted to the region of the muscle closest to the nerve trunk. Other studies (L. Landmesser and H. Tanaka, unpublished observations) have shown that this is the region of the muscle which contains the most mature myotubes as indicated by myosin ATPase staining. (B) The same muscles by stage 31 have fully cleaved (note low NCAM immunoreactivity between muscles) and most myotubes in both muscles stain intensely for NCAM. The peroneal trunk of the sciatic is still adjacent to the IF; the tibial trunk is no longer within the picture. Calibration bar $=200 \mu \mathrm{m}$. 

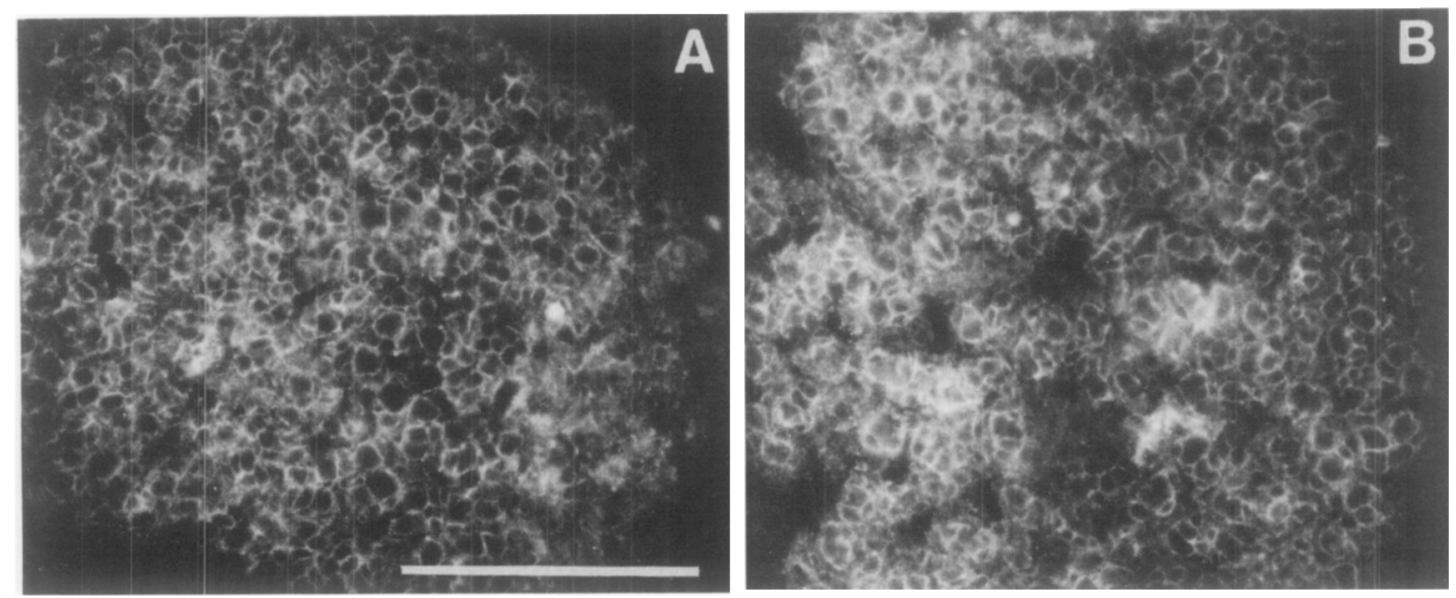

FIG. 7. NCAM expression on aneural myotubes. Transverse sections through a portion of the iliofibularis muscle at stage 34 from (A) a control embryo and (B) one whose neural tube had been removed prior to axon outgrowth. Intense NCAM immunoreactivity can be seen to outline the myotubes in both muscles, there being no obvious difference in the timing of onset or intensity of NCAM expression in aneural muscles. Calibration bar $=100 \mu \mathrm{m}$.

on slow adductor muscle fibers at stage 45 , at both stages there is a clear difference in NCAM staining between the two heads of the adductor. By stage 45, NCAM immunoreactivity is already markedly diminished in the fast adductor. However, some other fast muscles (i.e., posterior iliotibialis) still had moderate levels of staining at this time. By 3 days posthatching, staining on all fast myotubes had declined to undetectable levels, whereas slow muscles exhibited weak but obvious staining.

With the exception of fast and slow muscle fibers described above, there was within a muscle no obvious correlation between the decline in staining for NCAM and the age of a given muscle fiber. In a fast muscle, such as the posterior iliotibialis, NCAM immunoreactivity declined from all muscle fibers more or less synchronously. Thus, primary myotubes which were present at stage 30 exhibited a decrease in staining for NCAM at the same time as secondary myotubes which were generated a week or more later.

\section{Electrophoretic Properties of NCAM from the Developing Limb}

These studies were carried out to confirm biochemically the presence of NCAM in the tissues which were studied by immunocy tochemistry and also to determine the form of NCAM in the limb system during several developmentally important time periods. Both muscle and nerve tissue were dissected from embryos at different stages and immunoblots were prepared as described under Materials and Methods. In Fig. 10, immunoblots from these tissues (Figs. 10g-k) can be compared with the more heavily sialated brain NCAM (Figs 10a and b), and the less heavily sialated forms in breast muscle (Figs. 10c and d), and retina (Figs. 10e and f).
By stages 28-30, neuronal growth cones have grown into the limb and selected appropriate muscle nerves with considerable precision but have not yet ramified within the muscle to any extent (Tosney and Landmesser, 1985a). Neuronal or "plexus" NCAM appears at this stage as a diffuse band extending from 140,000 to 200,000 $M_{\mathrm{r}}$ (Fig. $10 \mathrm{j}$ ), and is thus intermediate in migration between the more heavily sialated brain form (Figs. 10a and b) and the less heavily sialated muscle (Figs. 10c and d) or retinal (Figs. 10e and f) forms. There was no change in the form of plexus NCAM during the next few stages, when axons begin to diverage from each other and ramify within the developing muscles (Figs. 10i and j). By stage 40, however, when the major period of synapse formation is complete, plexus NCAM becomes less sialated. In addition to a distinct band at $140 \mathrm{kDa}$, the plexus NCAM also includes a $120-\mathrm{kDa}$ component which may be characteristic of NCAM from glial cells (Goridis et al., 1983).

As with nerve, muscle NCAM did not change appreciably in form between stages 28 and 30 (Fig. 10g) and stages 32 and 33 (Figs. $10 \mathrm{~g}$ and $\mathrm{h}$ ), and gave an electrophoretic pattern similar to 12-day (stage 38) breast muscle (Figs. 10c and d), with diffuse staining from 140,000 to $200,000 M_{\mathrm{r}}$ and a more intense band at about $140,000 M_{\mathrm{r}}$. Therefore the form of NCAM was similar before, during, and after innervation.

\section{DISCUSSION}

Our observations provide interesting correlations between the distribution of staining for NCAM and developmental events in limb innervation. While such correlations alone cannot establish causality we will discuss our observations as they relate to speculative yet testable 

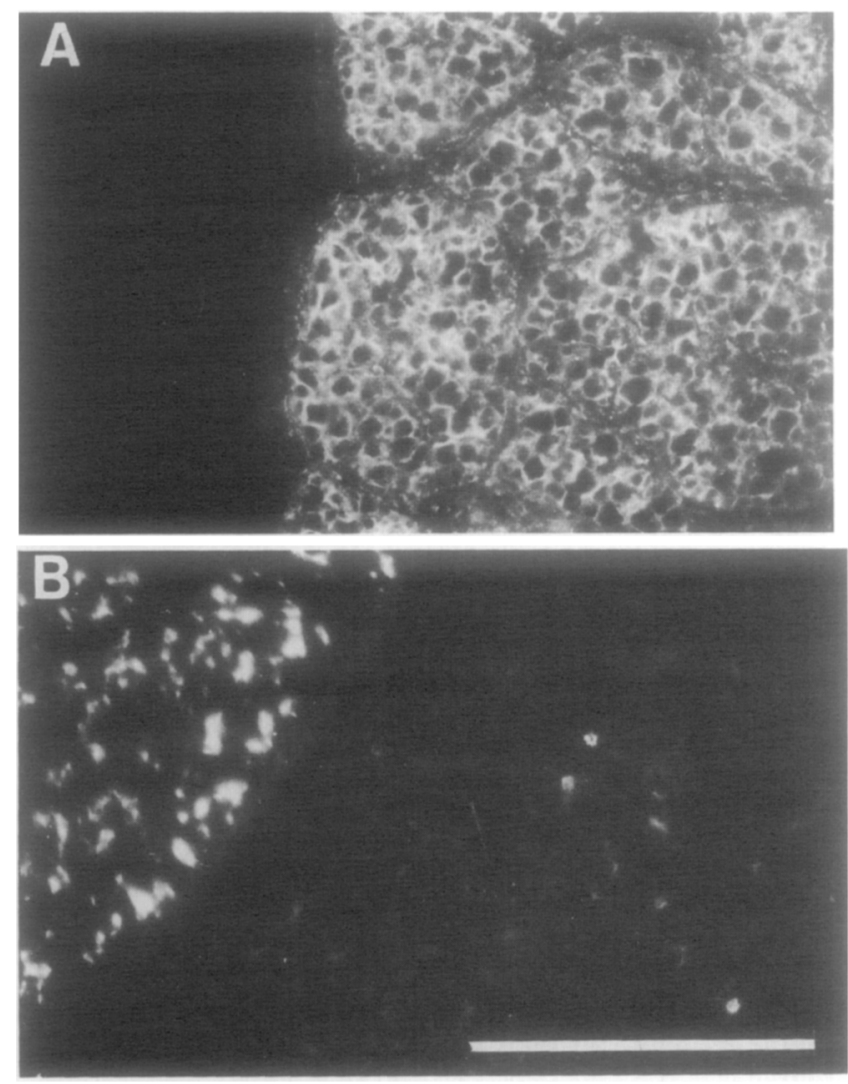

FIG. 8. The decline of NCAM expression on muscle fibers at hatching. (A) A cross section through the posterior iliotibialis muscle at stage 40 shows intense staining for NCAM on most myotubes. (B) A transverse section through a portion of the same muscle at 3 days posthatching shows weak to absent staining for NCAM on myotubes except for a small number of intensely stained nerve terminals. A portion of an intramuscular nerve branch on the left shows intense NCAM immunoreactivity of axons. Calibration bar $=100 \mu \mathrm{m}$.

hypotheses of how NCAM may function in each of five events that appear to contribute to the establishment of chick hindlimb innervation: (a) growth from the spinal cord to the plexus region, (b) specific deployment of axons in the plexus region and where muscle nerves diverge, (c) fasciculation to form nerve trunks, (d) ramification of axons within the target, and (e) stabilization of synaptic interactions.

\section{Pathway Guidance}

It has been suggested that a system of pathways exists within the vertebrate limb that are preferred substrata for axonal elongation (Lance-Jones and Landmesser, 1981b; Lewis et al., 1981; Tosney and Landmesser, 1984, $1985 \mathrm{~b})$. These pathways would define the gross anatomical pattern of nerve outgrowth and lead growth cones into regions where they could respond to specific cues. If NCAM alone delineates these pathways, we would expect it to appear within the pathways before nerve out- growth and to be absent or at reduced levels in adjacent axon-refractory regions, as has been observed in the chick retinotectal system (Silver and Rutishauser, 1984). By these criteria, our observations suggest that while NCAM may be necessary for axon outgrowth and control the temporal sequence in which axons leave the cord it does not by itself precisely define substrate pathways.

Keynes and Stern (1984) have shown that the earliest axons prefer to grow through the anterior portion of each somite, and that it is the somite that imposes the segmental pattern of spinal nerves. We have not observed any clear differences in NCAM staining within each somite correlated with this preference. Similarly, within the limb, we did not observe an increased inten-
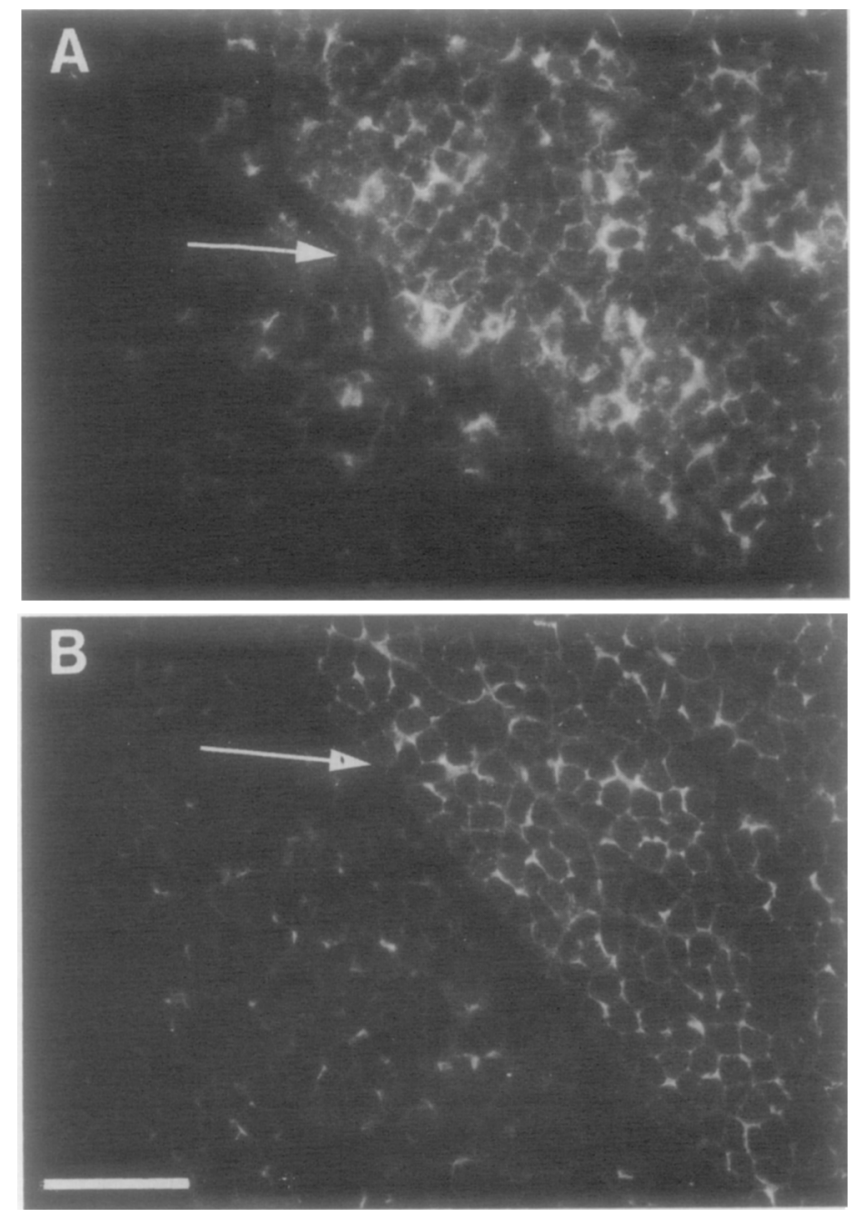

FIG. 9. Temporal differences in the decrease of NCAM on fast and slow muscle fibers. The border (indicated by arrows) between the slow external (right) and fast internal (left) adductor muscles runs diagonally across these transverse sections at (A) stage 45 and (B) 3 days posthatching. At both stages, the entire circumference of most of the muscle fibers in the slow adductor exhibits moderate immunoreactivity for NCAM, whereas such staining is reduced or absent on the muscle fibers of the fast muscle. More intense patches of immunoreactivity for NCAM in both muscles probably correspond to synaptic regions. Calibration bar $=200 \mu \mathrm{m}$ for $\mathrm{A}, 100 \mu \mathrm{m}$ for $\mathrm{R}$. 


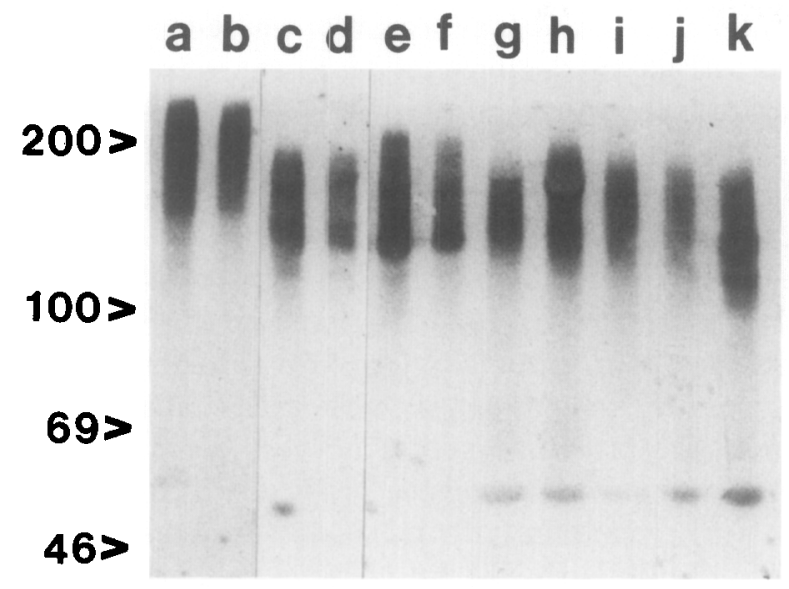

FIG. 10. Electrophoretic properties of NCAM from chicken embryo tissues. The electrophoretic mobility in sodium dodecyl sulfate-polyacrylamide gel electrophoresis (SDS-PAGE) of NCAM from aneural limb muscle from stages 28 to $30(\mathrm{~g})$ and $35 \frac{1}{2}(\mathrm{~h})$ embyros were similar to each other and to that of NCAM from breast muscle (c, d) and retinal tissue $(e, f)$. There is no evidence for changes in the form of NCAM in limb muscle during the stages when immunohistological staining intensities suggest an increase in the amount of NCAM. The properties of NCAM from plexus at stages 28-30 (i) and 32-33 (j) were also similar, appearing as a broad band at the same $M_{r}$ range as limb muscle NCAM but without intense bands. By stage 40 , however, the NCAM from the plexus (k) migrated in a lower $M_{r}$ range and included several discrete bands, one at $120,000 M_{\mathrm{r}}$ and another at $140,000 M_{\mathrm{r}}$. Brain NCAM (a, b) migrated as a broad, diffuse band at a higher $M_{r}$ range than muscle, retina, or plexus and exhibited no intensely stained bands. The component at $50,000 M_{r}$ is the monoclonal antibody which is released from the beads during the sample solubilization for SDSPAGE and recognized by the cross-reacting, ${ }^{125}$ I-labeled goat antirabbit second antibody. It is present when antibody-coated beads alone are processed for SDS-PAGE (data not shown). The molecular weight standards were myosin $200,000 M_{r}(200)$, phosphorylase B $100,000 M_{r}$ (100), bovine serum albumin $69,000 M_{\text {; }}(69)$, and ovalbumin $46,000 M_{\mathrm{r}}$ (46). Distortion of the pattern in the $180,000-200,000 M_{\mathrm{r}}$ region of some samples is due to contamination with myosin.

sity of staining for NCAM along prospective pathways in advance of the nerve front. In particular, we saw no differential distribution in NCAM immediately distal to the plexus region at stages $20-24$ that could explain the delay in axonal advancement that occurs at this point between stages 20 and 23 (Tosney and Landmesser, 1985b). However, in both the sclerotome and the limb NCAM was present within all regions through which axons grew. It will be interesting to determine if it is actually required for axon outgrowth, a possibility that we plan to test by applying function-blocking antibodies.

Further, while the adhesive function and tissue distribution of NCAM suggests that this molecule may be necessary for the growth of axons into the limb, it is probable that other factors are also involved. For example, in an analogous system, fibronectin is a preferred substratum for neural crest cells in culture (Thiery et $a l ., 1982)$; yet, fibronectin alone does not precisely delin- eate crest cell pathways in the chick embryo, since it is present along a pathway lateral to the neural tube in the mesencephalon which crest cells do not colonize (see Fig. 5C in Duband and Thiery, 1982). Moreover, in addition to fibronectin "interstitial bodies," ultrastructurally identifiable complexes of fibronectin, collagen, and glycosaminoglycans (Hay, 1978), are distributed congruently with all early neural crest pathways in the chick, are contacted by crest cell filipodia, and disappear following migration (Tosney, 1978, 1982, and unpublished observations). Similarly, in the chick limb both laminin (Rogers et al., 1984) and NCAM appear to be present within the pathways motoneurons follow and each is a good substrate for growth cones in vitro (Rogers et al., 1983; Rutishauser and Edelman, 1980). In addition, the restriction of axons within pathways is likely to be influenced by the presence of adjacent, less adhesive substrate. For instance, large amounts of glycosaminoglycans, on which growth cones extend poorly in vitro (Carbonetto et al., 1983) have been detected with Alcian blue staining (Tosney and Landmesser, 1985b) ventral to the spinal nerve pathway as well as immediately distal to the plexus in a region shown experimentally to be a barrier to axonal outgrowth (Tosney and Landmesser, 1984).

\section{Specific Deployment of Axons in the Plexus Region}

Specific guidance cues must also be postulated to explain the precise deployment of motoneuron axons in regions where substrate pathways branch. During normal development, motoneurons deploy with great precision in two such locations: the plexus region (LanceJones and Landmesser, 1981a) and the regions where muscle nerves diverge from nerve trunk pathways (Tosney and Landmesser, 1985b).

Although NCAM is present at low to moderate levels on the mesenchymal cells of the plexus, there is no evidence to suggest that this molecule is expressed in a manner that could specify directly the appropriate rearrangement of axons according to their eventual targets that takes place in the plexus (Lance-Jones and Landmesser, 1981a). Therefore, as in the case of tectal cues for optic axons in the chick visual system (Silver and Rutishauser, 1984), we propose that NCAM may help to bring axons to the region where positional cues are expressed, but does not (except possibly in a supportive role) participate in their specific guidance.

\section{Fasciculation of Nerves and Growth to Appropriate Muscles}

NCAM is expressed on all motoneurons, and the ability of this molecule to promote the formation of nerve bundles has been documented both in vitro (Rutishauser et al., 1978) and in vivo (Thanos et al., 1984). Two sets of 
observations suggest that strong interneuronal adhesion may serve to spatially delimit the response of axons to specific cues. When the limb bud is rotated about the dorsal-ventral axis and the rotation is quite proximal (i.e., at the plexus level), axons are able to alter their trajectory and project to correct targets (Ferguson, 1983). However, when rotations are made more distally, at a point where axons have already sorted out into nerve trunks, axons appear unable to sense their appropriate displaced targets and project indiscriminately to foreign muscle (Whitelaw and Hollyday, 1983b). Strong adhesions among neurites may thus prevent axons from responding to specific environmental cues after leaving the plexus, and thereby from compensating after certain experimental manipulations (cf, Landmesser, 1984; Tosney and Landmesser, 1985a).

We did not observe obvious differences in the intensity of staining for NCAM on nerves in the plexus versus the nerve trunk. However, the plexus is a loose tissue composed of small fascicles of axons interspersed among mesenchyme cells that stain only weakly for NCAM. At more distal levels where nerve trunks have formed, most axons are surrounded by other axons and this proximity alone would be expected to enhance fasciculation ( $\mathrm{Ru}$ tishauser and Edelman, 1980) and thereby promote the maintenance of fiber positions established in the plexus.

A high degree of adhesive interaction among the axons in this system is also suggested by the flattened, lamellepodial spreading of their growth cones on other neurites (Tosney and Landmesser, 1985a; see also Al-Ghaith and Lewis, 1982). The process of fasciculation, in part mediated by NCAM, may enhance the consolidation of axons into discrete spinal nerves and nerve trunks, preserve the sorting out of axons achieved in the plexus region by assuring that like axons remain together, and prevent premature divergence of axons from the nerve. Thus, while possibly acting primarily in a permissive manner, NCAM-mediated neuronal fasciculation could contribute to the specificity of the final innervation pattern.

We also observed that sensory axons stain less intensely for NCAM than do motoneurons during early stages of outgrowth. This differential distribution may simply reflect the fact that NCAM expression is altered in sensory neurons during their period of migration in the neural crest (Thiery et al, 1982). It could nevertheless also affect subsequent choices made by sensory growth cones (Honig, 1982) and may contribute to observed differences between sensory and motoneuron growth cones in both morphology and behavior. Although both types of axons grow out at approximately the same time, sensory growth cones are on the average considerably smaller and less lamellepodial than motoneuron growth cones (Tosney and Landmesser, 1985c). Furthermore, when motoneurons are surgically removed prior to axon outgrowth, sensory axons appear to fasciculate less strongly in the plexus (i.e., spinal nerves converge only briefly and there appears to be minimal exchange of axons between them). Finally, in the absence of motoneurons, sensory neurons fail to project down muscle nerves (Landmesser and Honig, 1986). There is therefore a clear difference in the responses of sensory and motoneuron growth cones to cues within the limb bud mesenchyme. The extent, if any, to which these differences in growth cone behavior are caused by differences in levels of NCAM remains to be determined.

\section{Ramification within the Target}

The increase in the intensity of staining for NCAM on myotubes is closely correlated with the ramification of axons within the early muscle masses. Although axons leave the major nerve trunks at stages $26-26 \frac{1}{2}$ and project into the uncleaved primary muscle masses, it is only at stages 30-31, some $48 \mathrm{hr}$ later, that individual axons diverge substantially from the main muscle nerves and begin to form small intramuscular nerve branches. In the intervening period, most motoneuron growth cones are found in contact with other axons within the major muscle nerves (Tosney and Landmesser, 1985a). It would therefore appear that during this "waiting" period, growth cones find contact with other axons preferable to contact with the surrounding mesenchyme, which at this stage is composed of differentiating myotubes with relatively low amounts of NCAM. A large increase in the amount of NCAM on the surface of myotubes occurs between stages 28 and 32, and may alter the growth cones' adhesive preferences so that myotubes become an effective substratum for axonal growth. Thus the growth cones would be able to leave muscle nerves, ramify within the muscle mass, and form synapses. It is of course possible that the correlation that we have observed is fortuitous and that the egress of axons from the muscle nerve trunks is caused by other factors such as the secretion of trophic or neurite-inducing factors by the developing myotubes. Nevertheless, it is noteworthy that in contrast to limb axons, which pause before invading limb muscles, the myotomal axons enter the myotome with no apparent delay, forming elaborate and highly branched growth cones (Tosney and Landmesser, 1985a,c). The cells composing the inner border of the myotome, which will differentiate into axial musculature, stain intensely for NCAM from the time that axons first leave the spinal cord.

NCAM does not appear to play a direct role in the specific guidance of motor axons in the plexus. Nevertheless, the delay in axon ramification within the muscle masses could contribute indirectly to the speci- 
ficity of muscle innervation, simply by preventing the approach of growth cones to presumptive boundaries between muscles until after muscle cleavage is complete. After such cleavages, growth cones would encounter clear physical boundaries, which do not contain high levels of NCAM, between their own and foreign muscles, and might therefore be restricted to their own muscle. It was in fact interesting that even within muscles, axon terminals seemed to be restricted to those regions of the muscle which contained myotubes with intense reactivity for NCAM.

NCAM could conceivably play a role in muscle cleavage itself by being differentially expressed within the premuscle masses. We did not observe a clear diminution in NCAM staining on mesenchyme cells that would predict where cleavage planes were going to form. However, since staining of mesenchyme cells was low to moderate, an actual decrease on cells along boundary regions might have gone undetected. A second way that NCAM could be involved would be if there is a nucleation phenomenon such that clusters of myotubes differentiate within one area of a prospective muscle and accretion of new myotubes occurs progressively up to the cleavage plane. It is clear from myosin ATPase staining of stage 27-30 limbs that initial differentiation of myotubes is clustered (L. Landmesser and H. Tanaka, unpublished observations). Nevertheless, individual primary myotubes at these stages are separated from each other by extensive extracellular space, making it difficult to envision how NCAM-mediated adhesions could contribute to this accretion process.

\section{Synaptic Interactions}

Although the enhancement of NCAM expression on myotubes does not require the presence of nerves, as we have shown that it occurs with a similar time course in aneural limbs, several observations suggest that nervemuscle interactions are important in the subsequent regulation of this molecule. Recently, Couvalt and Sanes (1985) and Rieger et al. (1985) have shown that NCAM is present in high amounts on embryonic myotubes in the mouse and that it decreases to undetectable levels on mature muscle fibers except at end plates, but reappears following denervation. Thus the low level of NCAM on muscle in adult mouse does appear to be dependent on neuron-muscle interaction, presumably mediated by activity or a trophic substance.

We have found that the intensity of staining for NCAM remains high on myotubes throughout the period of synapse formation (Landmesser and Morris, 1975; Landmesser, 1978; Bekoff, 1976) and only begins to decline during the withdrawal of polyneuronal innervation (Bennett and Pettigrew, 1974; Srihari and Vrbova, 1978;
Pockett, 1981). Since the muscle basal lamina has not formed at the time of initial synapse formation (Hirano, 1967; Kikuchi and Ashmore, 1976), the nerve terminal would come into direct contact with the surface of the myotube. NCAM on the surface of myotubes may thus play an important role in synapse formation onto primary myotubes and onto secondary myotubes as they are generated (see Rutishauser et al., 1983, for relevant observations in culture). A role for NCAM during reinnervation or during the withdrawal of polyneuronal innervation is less easy to visualize, since a well-formed basal lamina separates NCAM on the myotube surface from the nerve terminal (cf, Nitkin et al., 1983). However, NCAM at other sites, for example on glial cells or even in the basal lamina itself, could be involved.

As has already been observed for the mammal, we found that, around the time of hatching, NCAM expression on muscle declined dramatically, remaining intense only along axons and at presumed endplates. In addition, we observed that the decline in NCAM expression was asynchronous, occurring first on fast muscle fibers and then on slow. In most animals, including birds (Srihari and Vrbova, 1978), slow and fast muscle fibers are activated with different patterns of activity, and in the chick embryo, individual motor units exhibit clear differences in activation patterns as early as stage 36 (O'Donovan, 1984). Activation patterns are known to differ in mature muscles and are responsible for a number of phenotypic differences between fast and slow muscle fibers, including the speed of contraction and form of myosin (for review, see Jolesz and Sreter, 1981). Thus one possibility is that NCAM expression is sensitive to the pattern of muscle fiber activation that is imposed by the fast or slow nature of the innervating motoneurons. However, it is somewhat puzzling that within either the fast or slow population of muscle fibers, NCAM immunoreactivity appeared to decrease on primary myotubes, which had been innervated and activated from stages 30-32 (Day 7) at approximately the same time as on secondary myotubes which had formed much later (stages $36-38$, days $10-12$ ). It is therefore possible that around hatching there is some overall change in the amount or pattern of muscle fiber activation which triggers the decline. Alternatively, a neuronal trophic signal or hormonal change may also be involved. It will now be of interest to determine if the abolition of nerve activity by pharmacological means can alter the different time courses in the decline of NCAM expression on fast or slow muscle fibers.

In summary, the distributions of NCAM in space and time in the chick limb have led us to propose multiple distinct roles for this molecule in axon outgrowth and synapse formation. These hypotheses make specific predictions which can now be tested by altering the function 
of NCAM in the embryo at various stages with specific antibodies as originally carried out on the visual system (Thanos et al., 1984; Silver and Rutishauser, 1984). For example, our hypotheses would suggest that the timely disruption of NCAM function could cause motor or sensory nerve outgrowth from the spinal cord to be abolished, delayed, more diffuse, or topographically abnormal. Similarly, disruption of adhesive interactions both in regions where specific deployment takes place and in the nerve trunk would be expected to result in less precise innervation. Finally, the inhibition of interactions between the muscle nerve and target may prevent or delay the ramification of growth cones within the target and thereby alter the normal patterns of synapse formation and stabilization.

We thank Sara Putnam for her secretarial assistance. This work was supported by NIH Grants NS 19640, HD 18369, NS 21308, and a Rackham Faculty Grant, University of Michigan.

\section{REFERENCES}

AL-Ghaith, L. K., and Lewis, J. H. (1982). Pioneer growth cones in virgin mesenchyme: An electron-microscope study in the developing chick wing. J. Embryol. Exp. Morphol 68, 149-160.

BEKUFF, A. (1976). Ontogeny of leg motor output in the chick embryo: A neural analysis. Brain Res. 106, 271-291.

RfNnetr, M. R., and PFitrigrew, A. G. (1974). The formation of synapses in striated muscle during development. J. Physiol. (London) 241, 515-545.

Bentley, D., and CAUDY, M. (1983). Navigational substrates for peripheral pioneer growth cones: Limb-axis polarity cues, limb-segment boundaries and guidepost neurons. Cold Spring Harbor Symp. Quant. Biol. 48, 573-585.

Bentley, D., and Keshishian, H. (1982). Pathfinding by peripheral pioneer neurons in grasshoppers. Science (Washington, D. C.) 218, 1082-1088.

BERLOT, J., and GOODMAN, C. S. (1984). Guidance of peripheral pioneer neurons in the grasshopper: Adhesive hierarchy of epithelial and neuronal surfaces. Science (Washington, D. C.) 223, 493-496.

BRAY, D. (1982). Filipodial contraction and growth cone guidance. In "Cell Behavior" (R. Bellairs, A. Curtis, and G. Dunn, eds.), pp. 299318. Cambridge Univ. Press, London/New York.

Brown, M. C., JAnsen, J., and Van Essen, D. (1976). Polyneuronal innervation of skeletal muscle in new-born rats and its elimination during maturation. J. Physiol. (London) 261, 387-402.

Carbonnetto, S., Gruver, M. M., and Turner, D. C. (1983). Nerve fiber growth in culture on fibronectin, collagen, and glycosaminoglycan substrates. J. Neurosci. 3, 2324-2335.

ChU-Wang, I.-W., and OPPenheim, R. W. (1978). Cell death of motoneurons in the chick embryo spinal cord. II. A quantitative and qualitative analysis of degeneration in the ventral root, including evidence for axon outgrowth and limb innervation prior to cell death. J. Comp. Neurol. 177, 59-86.

Couvalt, J., and SANES, J. (1985). Neural cell adhesion molecule (NCAM) accumulates in denervated and paralyzed skeletal muscle. Proc. Natl. Acad. Sci. USA, 82, 4544-4548.

DUBAND, J. L., and THIERY, J.-P. (1982). Distribution of fibronectin in the early phase of avian cephalic neural crest cell migration. Dev. Biol. 93, 308-323.

FERGUSON, B. A. (1983). Development of motor innervation of the chick following dorsal-ventral limb bud rotations. J. Neurosci. 3, 17601772.

Goridis, C., Deagostini-Bazin, H., Hirn, M., Hirsch, M.-R., Rovgon, G., Sadoul, R., Langley, O. K., Gombos, G., and Finne, J. (1983). Neural surface antigens during nervous system development. Cold Spring Harbor Symp. Quant. Biol. 48, 527-537.

Grumet, M., Rutishauser, U., and Edelman, G. M. (1982). Neural cell adhesion molecule is on embryonic muscle cells and mediates adhesion to nerve cells in vitro. Nature (London) 295, 693-695.

Hamburger, V. (1939). The development and innervation of transplanted limb primordia of chick embryos. J. Exp. Zool. 80, 347-385.

HAMBURGER, V. (1975). Cell death in the development of the lateral motor column of the chick embryo. J. Comp. Neurol. 160, 535-546.

HAMBURGER, V., and HAMILTON, H. L. (1951). A series of normal stages in the development of the chick embryo. J. Morphol. 88, 49-82.

HAY, E. D. (1978). Fine structure of embryonic matrices and their relation to the cell surface in rutheium red-fixed tissues. Growth $\mathbf{4 2}$, 399-423.

HiRANo, H. (1967). Ultrastructural study on the morphogenesis of the neuromuscular junction in the skeletal muscle of the chick. Z. Zellforschung. Mikrosk. Anat. 79, 198-208.

HOLLYDAY, M. (1981). Rules of motor innervation in chick embryos with supernumerary limbs. J. Comp. Neurol. 202, 439-465.

HOLLYDAY, M. (1983). Cell and tissue interactions in the organogenesis of the avian limb musculature. In "Limb Development and Regeneration" (J. F. Fallon and A. I. Caplan, eds.), Part B, pp. 293-302. Liss, New York.

HoNIG, M. (1982). The development of sensory projection patterns in embryonic chick hind limb. J. Physiol. (London) 330, 175-202.

Johnson, D. A., GAUTSCH, J. W., and SprotsMan, J. R. (1984). Improved technique utilizing nonfat dry milk for analysis of protein and nucleic acid transferred to nitrocellulose. Gene. Anal. Tech. 1, 3-8.

KATZ, M. J., and LASEK, R. J. (1979). Substrate pathways which guide growing axons in Xenopus embryos. J. Comp. Neurol. 183, 817-832.

Keynes, R. J., and Stern, C. D. (1984). Segmentation in the vertebrate nervous system. Nature (London) 310, 786-789.

KIKUCH, T., and ASHMoRe, C. P. (1976). Developmental aspects of the innervation of skeletal muscle fibers in the chick embryo. Cell Tissue Res. 171, 233-251.

Jolesz, F., and SRETER, F. A. (1981) Development, innervation, and activity induced changes in skeletal muscle. Ann. Rev. Physiol. 43, 531-552.

LAEMMLI, U. K. (1970). Cleavage of structural proteins during the assembly of the head of bacteriophage T4. Nature (London) 277, 680685.

LANCE-JONFs, C., and LANDMESSFR, L. (1980a). Motoneurone projection patterns in embryonic chick limbs following partial deletions of the spinal cord. J. Physiol. (London) 302, 559-580.

LANCE-JONES, C., and LANDMESSER, L. (1980b). Motoneurone projection patterns of the chick hind limb following early partial reversals of the spinal cord. J. Physiol. (London) 302, 581-602.

LANCE-JONES, C., and LANDMESSER, L. (1981a). Pathway selection by chick lumbosacral motoneurons during normal development. Pror. R. Soc. London B 214, 1-18.

LANCE-Jones, C., and LANDMESSer, L. (1981b). Pathway selection by embryonic chick motoneurones in an experimentally altered environment. Proc. R. Soc. London B 214, 19-52.

LANDMESSER, L. (1978). The development of motor projection patterns in the chick hind limb. J. Physiol. (London) 284, 391-414.

LANDMESSER, L. (1984). The development of specific motor pathways in the chick embryo. Trends Neurosci. 7, 336-339.

LANDMESSER, L. and HoNIG, M. (1986). Altered sensory pathway selection in the chick limb following motoneuron removal. (submitted for publication) 
LANDMESSER, L., and MORRIS, D. G. (1975). The development of functional innervation in the hind limb of the chick embryo. J. Physiol. (London) 249, 301-326.

Letourneau, P. C. (1975). Cell-to-substratum adhesion and guidance of axonal elongation. Dev. Biol. 44, 92-101.

Lewis, J., Chevallier, A., Kieny, M., and WolPert, L. (1981). Muscle nerves do not develop in chick wings devoid of muscle. J. Embryol. Exp. Morphol 64, 211-232.

MCLENNEN, I. S. (1983). The development of the pattern of innervation in the chick hindlimb muscles: Evidence for the specificity of nervemuscle interaction. Dev. Biol. 97, 229-238.

NARDI, J. B. (1983). Neuronal pathfinding in developing wings of the moth Manduca sexta. Dev. Biol. 95, 163-174.

NitKIn, R. M., Wallace, B. G., SpIRA, M. E., Godfrey, E. W., and MCMAHAN, U. J. (1983). Molecular components of the synaptic basal lamina that direct differentiation of regenerating neuromuscular junctions. Cold Spring Harbor Symp. Quant. Biol. 48, 653-665.

O'DONOVAN, M. J. (1984). Firing patterns and recruitment behavior of single embryonic motoneurons. Soc. Neurosci. Symp. Abst. 10, 915.

POCKETT, S. C. (1981). Elimination of polyneuronal innervation in proximal and distal leg muscles of chick embryos. Dev. Brain Res. 1, 299-302.

Raper, J. A., Bastiani, M., and Goodman, C. S. (1983a). Pathfinding by neuronal growth cones in grasshopper embryos. I. Divergent choices made by the growth cones of sibling neurons. $J$. Neurosci. 3, 20-30.

Raper, J. A., Bastiani, M., and Goodman, C. S. (1983b). Pathfinding by neuronal growth cones in grasshopper embryos. II. Selective fasciculation onto specific axonal pathways. J. Neurosci. 3, 31-41.

Rieger, F., Grumet, M., and Edelman, G. M. (1985). N-CAM at the vertebrate neuromuscular junction. J. Cell Biol. 101, 285-293.

Rogers, S. L., Letourneau, P. C., Palm, S. L., McCarthy, J., and FuRCHT, L. T. (1983). Neurite extension by peripheral and central nervous system neurons in response to substratum-bound fibronectin and laminin. Dev. Biol. 98, 212-220.

Rogers, S. L., McLoon, S. C., and Letourneau, P. C. (1984). Distribution of laminin and fibronectin during early axonal growth in the chick PNS. Soc. Neurosci. Symp. Abst. 10, 39.

RUTISHAUSER, U. (1983). Molecular and biological properties of a neural cell adhesion molecule. Cold Spring Harbor Symp. Quant. Biol. 48, 501-514.

RUTISHAUSER, U. (1984). Developmental biology of a neural cell adhesion molecule. Nature (London) 310, 549-554.

RUTISHAUSER, U., and EDELMAN, G. (1980). Effects of fasciculation on the outgrowth of neurites from spinal ganglia in culture. J. Cell Biol. 87, 370-378.

Rutishauser, U., Gall, W. E., and Edelman, G. M. (1978). Adhesion among neural cells of the chick embryo. IV. Role of the cell surface molecule CAM in the formation of neurite bundles in cultures of spinal ganglia. J. Cell Biol. 79, 382-393.

Rutishauser, U., GRUMeT, M., and EdelmaN, G. M. (1983). NCAM mediates initial interaction between spinal cord neurons and muscle cells in culture. J. Cell Biol 97, 145-152.

SILVER, J., and RUTISHAUSER, U. (1984). Guidance of optic axons in vivo by preformed adhesive pathway on neuroepithelial endfeet. Dev. Biol. 106, 485-499.

SiNGER, M., NorDLANDER, R. H., and EGAR, M. (1979). Axonal guidance during embryogenesis and regeneration in the spinal cord of the newt: The blueprint hypothesis of neuronal pathway patterning. $J$. Comp. Neurol 185, 1-22.

SRIHARI, T., and VRBOVA, G. (1978). The role of muscle activity in the differentiation of neuromuscular junctions in slow and fast chick muscles. J. Neurocytol. 7, 529-540.

STRAZNICKY, C. (1983). The patterns of innervation and movements of ectopic hindlimb supplied by brachial spinal cord segments in the chick. Anat. Embryol. 167, 247-262.

Summerbell, D., and STIRLING, R. V. (1981). The innervation of dorsoventrally reversed chick wings: Evidence that motor axons do not actively seek out their appropriate targets. J. Embryol. Exp. Morphoh 61, 233-247.

SwANSTROM, R., and SHANK, P. R. (1978). X-Ray intensifying screens greatly enhance the detection by autoradiography of the radioactive isotopes ${ }^{82} \mathrm{P}$ and ${ }^{125} \mathrm{I}$. Anal Biochem. 86, 184-192.

TANAKA, H., and OBATA, K. (1984). Developmental changes in unique cell surface antigens of chick embryo spinal motoneurons and ganglion cells. Dev. Biol 106, 26-37.

Thanos, S., BonhoEffer, F., and Rutishauser, U. (1984). Fiber-fiber interaction and tectal cues influence the development of the chicken retinotectal projection. Proc. Natl. Acad. Sci. USA 81, 1906-1910.

Thiery, J.-P., Duband, J. L., Rutishauser, U., and EdelmaN, G. M. (1982). Cell adhesion molecules in early chicken embryogenesis. Proc. Nath Acad. Sci. USA 79, 6737-6741.

THOMPSON, W. (1983). Synapse elimination in neonatal rat muscle is sensitive to pattern of muscle use. Nature (London) 302, 614-616.

TOSNEY, K. W. (1978). The early migration of neural crest cells in the trunk region of the avian embryo: An ultrastructural study. Dev. Biol. 62, 317-333.

TOSNEY, K. W. (1982). The segregation and early migration of cranial neural crest cells in the avian embryo. Dev. Biol. 89, 13-24.

Tosney, K. W., and Landmesser, L. T. (1984). Pattern and specificity of axonal outgrowth following varying degrees of chick limb bud ablation. J. Neurosci. 4, 2158-2527.

TOSNEY, K. W., and LANDmESSER, L. T. (1985a). Specificity of motoneuron growth cone outgrowth in the chick hindlimb. J. Neurosci. 5, 2336-2344.

Tosney, K. W., and Landmesser, L. T. (1985b). Development of the major pathways for neurite outgrowth in the chick hindlimb. Dev. Biol. 109, 193-214.

TOSNEY, K. W., and LANDMESSER, L. T. (1985e). Growth cone morphology and trajectory in the lumbosacral region of the chick embryo. $J$. Neurosci. 5, 2345-2358.

Tosney, K., Watanabe, M., Landmesser, L., and Rutishauser, U. (1985). Distribution of NCAM in the chick hindlimb during axon outgrowth and the establishment of mature nerve-muscle relationships. Soc. Neurosci. Symp. Abst. 11, 174.

Towbin, H., Staehelin, T., and Gordon, J. (1979). Electrophoretic transfer of proteins from polyacrylamide gels to nitrocellulose sheets: Procedure and some applications. Proc. Nath Acad. Sci. USA 76, 43504354.

VAN ESSEN, D. C. (1982). Neuromuscular synapse elimination: Structural, functional and mechanistic aspects. In "Neuronal Development" (N. C. Spitzer, ed.). Plenum, New York.

VoGEL, M.; and LANDMESSER, L. (1986). Distribution of myosin ATPase fiber types in embryonic chick limb muscles innervated by foreign motoneurons. (submitted for publication)

WhITELA ${ }^{\prime}$, V., and Hollyday, M. (1983a). Position-dependent motor innervation of the chick hindlimb following serial and parallel duplications of limb segments. J. Neurosci. 3, 1216-1225.

WHITELAW, V., and HOLLYDAY, M. (1983b). Neural pathway constraints in the motor innervation of the chick hindlimb following dorsoventral rotations of distal leg segments. J. Neurosci. 3, 1226-1233. 